Pteridines

Vol. 2, 1990, pp. $45-46$

Short Communication

\title{
GTP-CH Activity in Bovine Retina
}

\section{Ludger Hanneken, Gertrud Cremer-Bartels, Heinrich Gerding and Kunibert Krause}

Klinik und Poliklinik für Augenheilkunde der Westfälischen Wilhelms-Universität, Domagkstr. 15, D-4400 Münster, Federal Republic of Germany

(Received March 1990)

\section{Introduction}

GTP serves as the substrate of GTP-CH I which catalyses the formation of dihydroneopterintriphosphate, the initial pteridine precursor for the biosynthesis of tetrahydrobiopterin. Dihydroneopterintriphosphate is as an intermediate also involved in monapterin biosynthesis. In rats GTP-CH is considered to be the rate-limiting enzyme in tetrahydrobiopterin biosynthesis (1). This is indicated by the parallel relationship between tetrahydrobiopterin content and GTP-CH activity in different rat tissues and the fact, that no neopterin could be detected.

We found in bovine retina apart from high levels of biopterin also remarkably high monapterin-concentrations while neopterin was very low. This ratio was even more shifted in human retina where monapterin could be determined as the main pterin (2).

Basing on these findings we assumed a yet unknown function of pterins in the retina apart from the importance of $\mathrm{BH}_{4}$ in neurotransmitter synthesis. Aubailly et al. (3) proposed for pterins a photosensitizer function comparable to that of flavines which involves Type I and II photosensitation. He considered radical formation, light-absorption in the near-ultraviolett and changes of the redox state in response to light as possible mechanisms for pterin mediated photoregulation. The eye provides a unique situation for such photochemical reactions. We started experiments determining the capacity of pterin biosynthesis by means of GTP-CH activity in bovine retina. High activities of GTP-CH are often found in tissues with high requirements for $\mathrm{BH}_{4}$. We were interested if the measured high pterin concentrations in neuroretina and retinal pigmentepithelium (RPE) are paralleled by high activities of this enzyme and wanted to compare the results with the known high liver enzyme activity.
The retina can generally be divided into the neuroretina with the rods and cones and the underlying pigment epithelium. This is a single layer of polygonal cells which is responsible for the maintenance of many retinal functions. In pigmented eyes it contains many melanosomes and melanin and absorbs most of the incoming light. Light adaption mechanisms of the retina are influenced by the functional status of the pigment epithelium. These adaptive mechanisms are influenced by retinal neurotransmitters and $\mathrm{BH}_{4}$ is involved in such regulations. The reported high retinal concentrations of pterins may also be of importance regarding the proposed pterin photoreactions mentioned above.

\section{Method}

We determined the GTP-CH activity separately in the neuroretina and retinal pigment epithelium using a method according to Duch and coworkers (4). We measured this activity up to now only in crude tissue extracts. Further purifications of the enzyme have to be done yet. Fresh retinae or pigment epithelium cells were homogenized and centrifuged. The supernatant was incubated with GTP for $30 \mathrm{~min}$, after oxidation neopterintriphosphate was dephosphorylated so that neopterin could be determined by HPLC. In every third to fifth experiment we did not add GTP to the reaction mixture.

\section{Results and Discussion}

GTP-CH activity in homogenates of bovine liver, neuroretina and retinal pigment epithelium are demonstrated in Figure 1. The enzyme activity in neuroretina is in the same range as in the liver. The liver as mentioned before is known to have a relatively high enzyme activity (4) if compared to other organs. 
A comparable requirement for pterin-synthesis of these two organs may therefore be assumed. Compared to the values in rat liver, where we found the same activity as Bellahsene et al. (5), it appears to be slightly lower, however not statistically significant. In retinal pigment epithelium GTP-CH-activity could be determined in a 7 -fold higher range. This result correlates to our determinations of pterin concentrations in bovine retina. If we did not add GTP to the reaction mixture neopterin was below detection limit in liver, neuroretina and RPE due to the small volume of the added tissue extract.

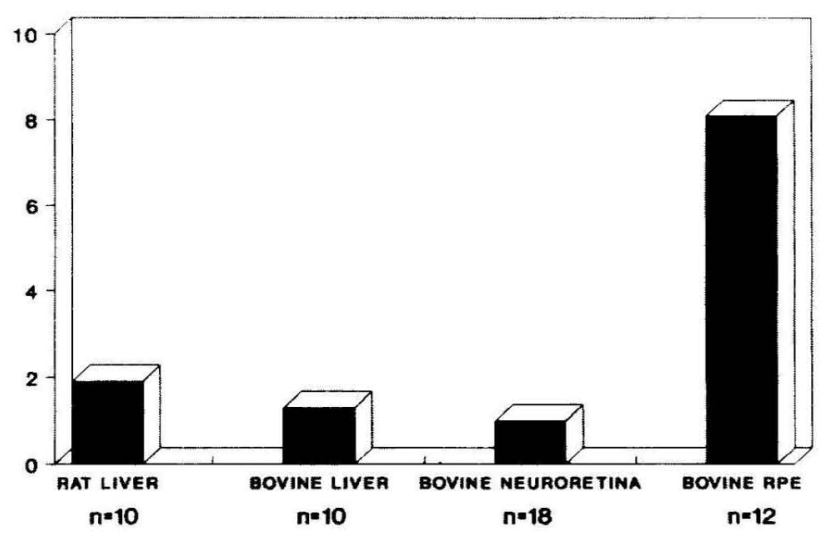

Figure 1. GTP-CH Activity in crude tissue extracts (ng neopterin $/ 30 \mathrm{~min} / \mathrm{mg}$ protein)

The neuroretina contains high pterin-concentrations compared to other tissues known in the literature. The RPE-concentrations of the determined pterins are in a much higher range. This difference between the two retinal parts is paralleled by GTP-CH activity which is about 7-times higher in the RPE than in the neuroretina. In bovine retinae biopterin is the main pterin. The necessity for this pterin may explain the high enzyme activity which we found.

While GTP-CH activity in neuroretina and liver is in the same range the ratio $\mathrm{NEO} / \mathrm{MON}$ however differs drastically (Fig. 2). While in liver the ratio is more than $4: 1$, it is inverse in the neuroretina with less than $1: 6$ with neopterin being sometimes below detection limit. This ratio was also found in pigment epithelium. Looking at the measured enzyme activities different levels of biopterin concentrations may be explained. The unusual NEO/MON ratio in neuroretina and RPE however may be based on a difference in the metabolism of dihydroneopterintriphosphate after being synthesized by GTP-CH. An enzyme activity that forms monapterin from neopterin may be required in a much higher activity in retinal tissues than in other ones. Considering our earlier reported results in human retina, where monapterin could be determined as the main pterin, such an enzyme activity would be of even greater importance. Unfortu- nately the difficulties in getting human material did not enable us yet to examine this problem. Alternatively an enzyme activity in humans or vertebrates which does not involve an epimerase that forms monapterin from neopterin could exist. A direct examination of such an epimerase in the retina or retinal pigment epithelium is necessary to clarify these problems. These experiments confirm our findings of high pterin concentrations in the retinae. They sustain our working hypothesis of a special importance of pterins for retinal physiology. Especially the unusual retinal pterin pattern with high monapterin levels is waiting for an explanation.

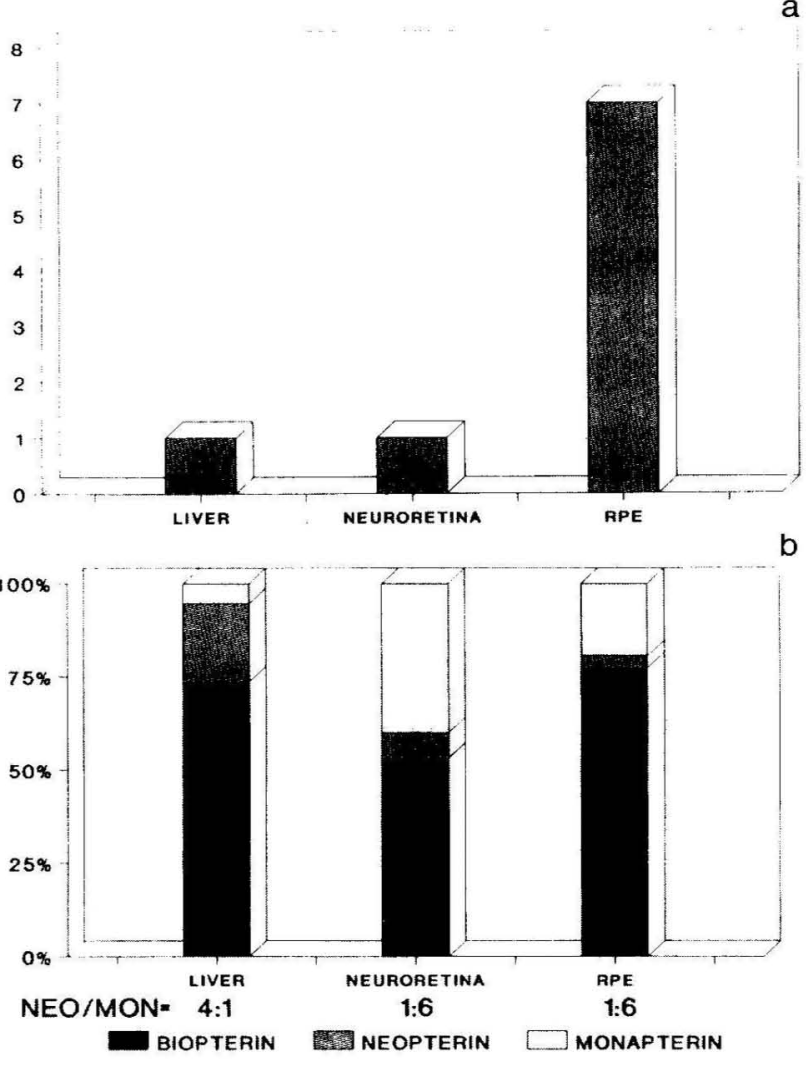

Figure 2. a) Relative GTP-CH activity

b) Pterin pattern in bovine tissue

\section{Acknowledgement}

This project is financially supported by the Deutsche Forschungsgemeinschaft AZ: Cr 40/11-1; Cr 40/11-2.

\section{References}

1. Viveros, O. H., Lee, C.-L., Abou-Donia, M., Nixon, J. C. \& Nichol, C. A. (1981) Science 213, 349-350.

2. Gerding, H., Cremer-Bartels, G., Hanneken, L. \& Krause, K. (1989) Biol. Chem. Hoppe-Seyler 370, 383.

3. Aubailley, M. \& Santus, R. (1986) In: Chemistry and Biology of Pteridines 1986 (Cooper, B. A. \& Whitehead, V. M., eds) pp. 99-103, Walter de Gruyter, Berlin, New York.

4. Duch, D. S., Bowera, S. W., Woolf, J. H. \& Nichol, C. A. (1984) Life Sciences 35, 1895-1901.

5. Bellahsene, Z., Dhondt, J. \& Farriaux, J. (1984) Biochem. J. $217,59-65$. 